\title{
Hydraulique pluviale à Nîmes après le 3 octobre 1988
}

\author{
Guilhem Fabre \\ Adjoint au Maire de Nîmes, Directeur de Recherche au C.N.R.S.
}

\author{
Jean-Marie Lequeux
}

Cabinet Merlin

Patrick Scheurer

Secrétaire Général Adjoint, Ville de Nîmes

Le phénomène hydropluvieux subi par Nîmes le 3 octobre 1988 s'est traduit par beaucoup de choses, dont 10 morts, 3 milliards de dégâts, une ville dévastée.

\section{Les caractéristiques de l'événement du 3 oc- tobre 1988}

\subsection{La météorologie}

Rappelons quelques hauteurs de précipitations : $226 \mathrm{~mm}$ à Nîmes-Courbessac, $310.5 \mathrm{~mm}$ à Nîmes-Kennedy, 220 à Nîmes Bas Rhône, $295 \mathrm{~mm}$ à Nîmes Puech du Teil, $420 \mathrm{~mm}$ au Mas de Ponge.

\subsection{Le contexte local}

La catastrophe du 3 octobre 1988 à Nîmes est le résultat de la convergence de 3 facteurs principaux :

- la topographie locale (concentration de collines au Nord du centre ville : chaque thalweg ou cadereau joue le rôle d'entonnoir et concentre les eaux de ruissellement vers le centre ville):

- la modification progressive des surfaces dans l'arrière pays provoquée par la désertification des collines et la déstabilisation des sols consécutives aux incendies de l'été :

- le développement urbanistique de la Ville : l'urbanisation des garrigues et la création de voies de communication augmentent l'imperméabilisation des surfaces - les infrastructures ferroviaires et routières en aval ont fait office de barrage aux eaux de ruissellement.

On est arrivé à la conclusion que 10 millions de $\mathrm{m}^{3}$ ont traversé la ville, charriant des matériaux de toute nature, gravois, barrières, voitures, mobilier, etc... sur les $14500000 \mathrm{~m}^{3}$ d'eau tombés sur l'ensemble des bassins versants de Nîmes (des pluies éparses les 3 jours précédents avaient saturé le karst des hauts bassins-versants).

Si le phénomène fut particulièrement catastrophique. depuis, d'autres débordements se sont produits le long de l'un ou l'autre des cadereaux, par exemple: septembre 1989. octobre 1990 où à Courbessac on a enregistré $267 \mathrm{~mm}$ en $5 \mathrm{~h}$ (plus que le 3 octobre 1988), septembre 1992, en 1993 ( 22 juin et 25 août et dernièrement les 20-21 octobre 1994).

Il suffit de se souvenir de Vaison La Romaine en septembre 1992, de Pertuis en septembre 1993, pour se rappeler que nous ne sommes pas à l'abri d'une nouvelle catastrophe.

En 1986, le Cabinet d'Etudes Merlin avait déjà réalisé une étude sur le pluvial mettant en évidence les insuffisances du réseau. Cette étude avait commencé à recevoir un débit d'exécution ( 2 bassins de retenue amont étaient en cours de réalisation).

Dès le 16 octobre 1988, une commission hydraulique fut créée par Monsieur le Député Maire de Nîmes pour tirer les enseignements de la catastrophe.

\section{La commission hydraulique}

\subsection{Sa mission}

C'est un organe d'études et de réflexions placé sous l'autorité directe du Maire. II eut pour mission l'analyse du phénomène survenu le 3 octobre 1988 à partir d'enquêtes (plus de $100 \mathrm{~km}$ de laisses de crues ont été relevées), d'études (simulations informatiques) et de travaux confiés notamment à des spécialistes et des experts ; l'animation. la coordination et la synthèse de tous ces travaux : l'aide à la décision des autorités au travers de propositions de solutions techniques. 


\subsection{Méthode de travail}

Les travaux de cette Commission ont été organisés en s'inspirant de la méthodologie des Plans d'Exposition aux Risques Inondations (PERI), à savoir : collecte et analyse de données; cartographie du phénomène d'inondation ; approche de la vulnérabilité ; évaluation de l'aléa ; propositions de solutions techniques.

\subsection{Ses travaux}

Proposition d'un plan d'aménagement permettant de faire face ou de limiter les effets destructeurs d'un orage tel que celui du 3 octobre 1988 intégrant 3 types de solutions techniques : construction de barrages de retenue en amont immédiat de la Ville; réalisation de tunnels collectant les eaux au droit des cadereaux; aménagement des écoulements naturels.

\subsection{Ses conclusions}

La perception des risques et leur prise en compte par les Pouvoirs Publics est étroitement liée à la survenance des phénomènes.

On doit considérer qu'urbanisme et évacuation des eaux pluviales sont pour le site de Nîmes indissociables.

Il convient dès lors de différencier les ouvrages d'assainissement banal et ceux relevant de l'aménagement de la Ville et devant la protéger des phénomènes destructeurs ; l'organisation de l'espace et donc l'urbanisme, doit prendre en compte cette contrainte prioritaire.

Les solutions proposées ont un impact sur l'aménagement du bassin du Vistre, et réciproquement. Il est donc nécessaire d'harmoniser les deux projets.

La commission hydraulique a abouti à un plan, avalisé par la mission PONTON, dont la première phase a reçu l'acceptation de la part de Monsieur Brice LALONDE alors Secrétaire d'Etat à l'Environnement. Celui-ci demandait, toutefois, des compléments d'étude et une liaison plus complète avec les projets à l'aval de Nîmes (plaine du Vistre).

C'est ainsi qu'un comité de pilotage fut mis en place par Monsieur le Préfet du Gard.

Pour mémoire, rappelons que le rapport de la mission PONTON a toujours été pris en compte à Nîmes pour la suite des études. Il n'avait pas été « enterré ».

\section{Le Comité de Pilotage}

Le Comité de Pilotage avait pour mission de préciser les caractéristiques des ouvrages de protection et les coûts des ouvrages ; d'assurer de la cohérence technique et temporelle de l'ensemble; d'étudier l'impact des travaux et ouvrages sur l'environnement ; d'examiner comment l'on pourra assurer la coordination entre les différents maîtres d'ouvrage ; et enfin d'aborder le problème de l'alerte aux populations.

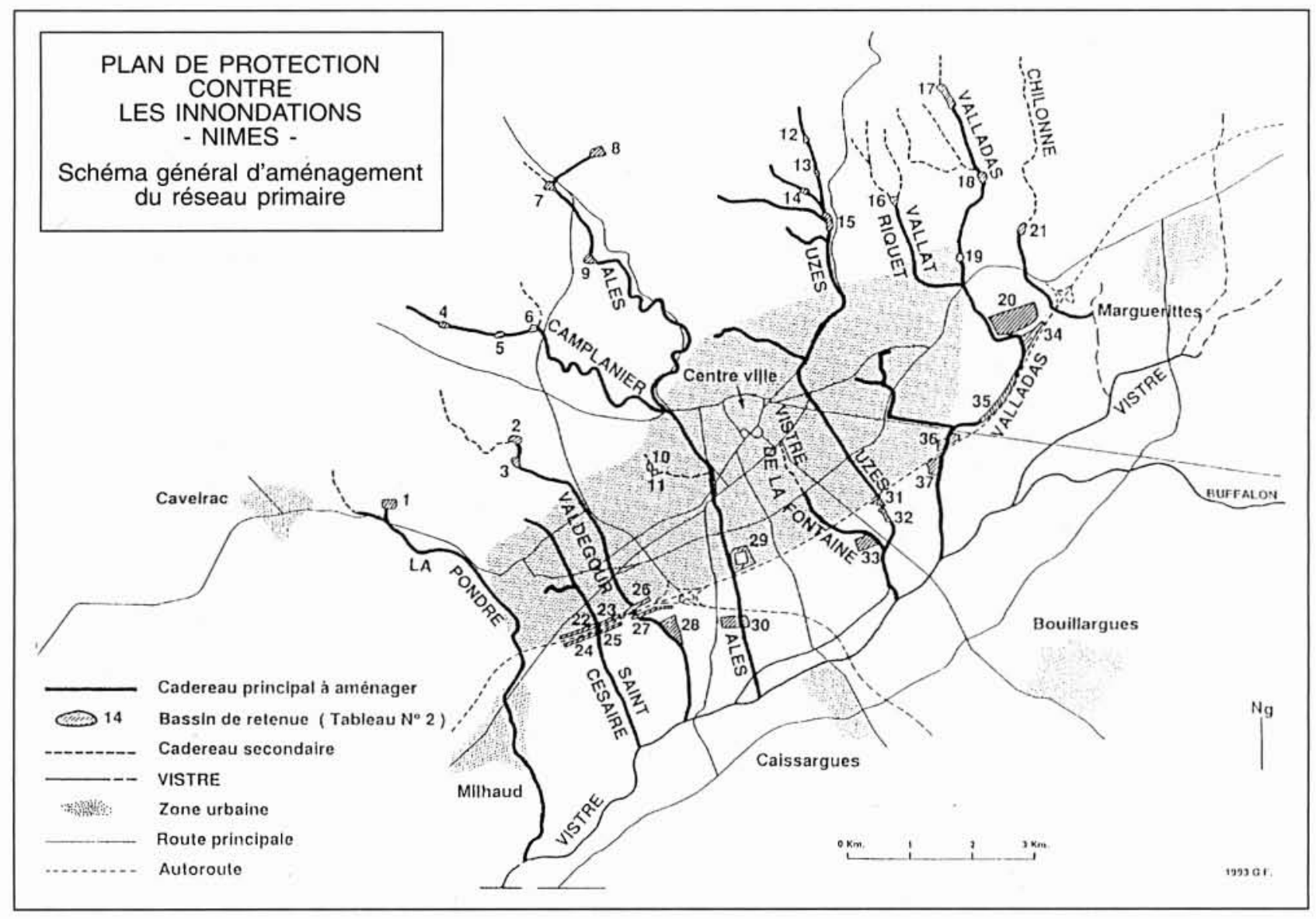


Ce Comité de Pilotage, à partir d'un cahier des charges établi par la Direction Départementale de l'Equipement, a confié cette étude au B.C.E.O.M.

Les conclusions ont été présentées au Comité de Pilotage le 26 novembre 1990.

A la suite de ces études préliminaires, on a pu établir le Plan de Protection Contre les Inondations (P.P.C.I.).

\section{Le P.P.C.I.}

Le P.P.C.I. ne concerne que le réseau primaire de la ville de Nîmes, c'est-à-dire l'ensemble des 8 cadereaux principaux qui la traversent.

Les objectifs de ce plan sont d'assurer la sécurité des Nîmois, de leurs biens ainsi que du patrimoine assurable ou non de la Ville quelles que soient les dispositions régissant la gestion du service et permettant la poursuite du développement économique de la Ville par la protection des zones sous influence des exutoires primaires contre des occurrences quarantennales à l'amont du boulevard Allende et centennales à l'aval de ce même boulevard (périphérique Sud).

Rappelons qu'une première solution dite des tunnels permettait de mettre la Ville de Nîmes à l'abri d'un phénomène pluvio-orageux du type 3 octobre 1988 .

Face à l'impossibilité de mobiliser son financement (2,5 milliards HT), la Ville a choisi de dégager une solution moins onéreuse permettant une protection homogène par bassin versant évaluée aujourd'hui à $670 \mathrm{MF}$.

Le schéma général proposé prévoit les travaux suivants :

\section{Bassins de retenue amont}

Création de Bassins répartis sur : La Pondre (1) dont une carrière de $5700000 \mathrm{~m}^{3}$ à Caveirac en cours de réalisation : Valdegour (2); Camplanier (4) dont 3 réalisés (2 à Vacqueyrolles et 1 au pont de la République) ; Alès (7) dont 1 carrière de $3000000 \mathrm{~m}^{3}$; Uzès (4): Vallat Riquet (1) réalisé ; Valladas (4) dont 2 réalisés (Caravanes et Aérodrome): Chilonne (1). Ces bassins représentent au total $9600000 \mathrm{~m}^{3}$ de stockage possible sur plus de 80 hectares et sont destinés à diminuer les débits pouvant traverser la ville au moment des grosses pluies.

Ils incluent un bassin dont le pertuis laisse écouler un débit limité vers l'aval (pour une pluie de 40 ans) et un déversoir pour les pluies d'occurrence supérieure. Les carrières stockent les eaux de ruissellement. Par pompage, après l'événement pluvieux, on réinjecte les eaux dans le réseau. Les carrières sont préférables aux bassins car elles constituent une protection totale (elles sont prévues pour pouvoir stocker 2 fois les quantités ayant transité le 3 octobre 1988 à leur endroit).

\section{Aménagement des cadereaux en amont de la ville}

Sur 8 cadereaux, aménagement de plus de $20 \mathrm{~km}$ de fossés de façon que les habitations soient protégées contre des pluies importantes (arrivant en moyenne tous les 40 ans) : La Pondre $1 \mathrm{~km}$; Saint-Césaire $1 \mathrm{~km}$; Valdegour 1,6 km ; Camplanier $3,2 \mathrm{~km}$ déjà réalisés ; Alès $6 \mathrm{~km}$; Uzès $3 \mathrm{~km}$; Vallat Riquet $1,2 \mathrm{~km}$; Valladas $3,6 \mathrm{~km}$.
L'ensemble des terrains nécessaires à ces travaux a été acheté par la ville.

Au titre du P.P.C.I., il n'est pas prévu de séparer dans la totalité des cadereaux la circulation des véhicules et celles des eaux de ruissellement.

\section{Traversée de la ville en souterrain}

Sur 6 cadereaux, aménagement de la traversée de la ville en souterrain jusqu'au boulevard périphérique, soit $13 \mathrm{~km}$ de canalisations allant de $2 \mathrm{~m}$ de diamètre à des cadres de $5,50 \mathrm{~m}$ de largeur et $3 \mathrm{~m}$ de hauteur: Valdegour $1,7 \mathrm{~km}$ : Alès $2,4 \mathrm{~km}$; chemin des Limites $2 \mathrm{~km}$; Uzès $3,5 \mathrm{~km}$ dont $1 \mathrm{~km}$ réalisé (rue des Résédas) ; Vallat Riquet $0,9 \mathrm{~km}$ dont $200 \mathrm{~m}$ réalisés (sous Intermarché): Mas Verdier $1,5 \mathrm{~km}$.

Ces cadereaux permettront ensemble de faire passer dans la ville près de $300 \mathrm{~m}^{3}$ par seconde alors qu'actuellement en passent moins de $80 \mathrm{~m}^{3}$ par seconde.

Une attention particulière est portée aux entonnements. Rien ne sert de faire des canalisations permettant de faire transiter le débit moyen de la Seine à Paris si on ne s'assure pas que l'eau y pénétrera en situation extrême.

\section{Fossés à l'aval du Boulevard Périphérique}

Entre la traversée de la ville en souterrain qui se passera en régime torrentiel et les fossés à ciel ouvert de l'aval dans lesquels les eaux seront en régime fluvial, il y a lieu de maîtriser le ressaut.

Environ $20 \mathrm{~km}$ de fossés sont et seront aménagés et en cours d'aménagement dans la plaine jusqu'au Vistre: Saint-Césaire $2,2 \mathrm{~km}$ (curage) : Valdegour $3,1 \mathrm{~km}$ (nouveau tracé); Alès $2,9 \mathrm{~km}$ (nouveau tracé); Vistre de la Fontaine $1,9 \mathrm{~km}$ (curage); Uzès $2,8 \mathrm{~km}$ (curage); Mas Verdier $1,1 \mathrm{~km}$ (nouveau tracé); Valladas $5,6 \mathrm{~km}$ (nouveau tracé).

Les anciens fossés seront bien entendu conservés et améliorés.

\section{Bassins de rétention à l'aval}

Création de 16 Bassins répartis sur Saint-Césaire (4), Valdegour (3), Alès (2), Uzès (1), Vistre de la Fontaine (1), Valladas (4) représentant plus d'un million de $\mathrm{m}^{3}$ de stockage et construits pour que les communes situées à l'aval de Nîmes ne reçoivent pas plus d'eau qu'auparavant dans les conditions du 3 octobre 1988.

Le calcul d'un indice d'efficacité/coût des travaux a conduit à établir une carte des priorités et a donc permis de déboucher sur un programme mettant en évidence la nécessité de réaliser en première urgence les bassins de retenues et de respecter la logique aval/amont pour la restructuration des réseaux.

\section{Législation administrative}

Le P.P.C.I. a été soumis à Enquête Publique.

Une première en 1992 a déclaré d'utilité publique le projet, sous réserve que l'on intègre les bassins aval. 
L'étude du Vistre faite par le B.C.E.O.M. étant achevée fin 1992, le P.P.C.I. a été soumis à une nouvelle enquête qui a abouti à une Déclaration d'Intérêt Général le 10 septembre 1993.

Le Préfet a demandé de coupler les enquêtes de D.U.P. et Hydrauliques, cadereau par cadereau.

Un premier dossier concernant le cadereau d'Uzès a été réalisé et présenté au public en avril 1994. Les autres dossiers suivent ( 1 tous les 3 mois environ).

Mais de façon à pouvoir avancer les travaux, des dérogations ont bien entendu été obtenues dès 1989 afin de réaliser les travaux les plus urgents.

\section{Financement}

Le financement de ce vaste projet, d'un coût voisin de $670 \mathrm{MF}$, se met en place : EDF, GDF, PTT modifient leurs réseaux, la SNCF apporte une aide de $52 \mathrm{MF}$, les ASF de $26 \mathrm{MF}$, l'Etat de $82 \mathrm{MF}$, la Région de $40 \mathrm{MF}$, l'Agence de Bassin $20 \mathrm{MF}$ et le Département $26 \mathrm{MF}$ pour ses ouvrages. Pour le reste, la Ville continue à rechercher des aides tout en investissant par elle-même la plus grande partie. Environ $82 \mathrm{MF}$ ont été engagés par la Ville à fin 1993. La programmation du reste du projet s'échelonne jusqu'à la fin du siècle et même au-delà.

\section{Récapitulation des dépenses par maître d'ouvrage (Francs HT, valeur novembre 1989)}

\begin{tabular}{|c|c|c|c|}
\hline & $\begin{array}{l}\text { Réalisé } \\
\text { fin } 1993\end{array}$ & Reste & TOTAL \\
\hline VILLE & 82 & 465,5 & 547,5 \\
\hline ETAT (RN) & 2,5 & 16 & 18,5 \\
\hline DEPARTEMENT (RD) & 4,2 & 21,8 & 26 \\
\hline A.S.F. (Autoroutes) & 0 & 26 & 26 \\
\hline S.N.C.F. & 0 & 52 & 52 \\
\hline TOTAL & 88,7 & 581,3 & 670,0 \\
\hline
\end{tabular}

\section{Subventions escomptées (Francs HT, valeur novembre 1989)}

\begin{tabular}{|c|c|c|c|}
\hline & A fin 1993 & Reste & TOTAL \\
\hline ÉTAT & 9,7 & 72,3 & 82 \\
\hline REGION & 2,5 & 137,5 & 140 \\
\hline DEPARTEMENT & 3,7 & 136,3 & 140 \\
\hline R.M.C. & 0 & 30 & 30 \\
\hline TOTAL & 15,9 & 376,1 & 392 \\
\hline
\end{tabular}

\section{Système d'alerte}

En complément du P.P.C.I., un système d'alerte contre les crues est en cours d'installation.

Les objectifs sont de :

- Protéger les populations contre les inondations brutales liées à des événements pluvio-orageux d'intensité exceptionnelle.

- Apporter la sérénité aux nombreuses familles et personnes âgées fortement traumatisées par les événements du 3 octobre 1988 .

- Analyser les formations de phénomènes pluvioorageux sur le bassin de Nîmes et leurs conséquences sur le terrain, afin d'améliorer à terme la prévention des risques.

\section{Dispositions régies par l'article $R$ 111-3 du Code de l'Urbanisme}

En complément du P.P.C.I., on a également mis en place des dispositions régies par l'article R 111-3 du Code de l'Urbanisme.

Très succinctement, la ville, séparée en 3 secteurs, est soumise aux règles suivantes:

Secteur 1 : la Garrigue.

Dans les cadereaux, les constructions sont interdites sous les laisses de crues du 3 octobre 1988.

Secteur 2: le Piémont.

Dans les zones diffuses, il est demandé de prévoir des bassins de rétention de 50 litres/mètre carré imperméabilisé.

Dans les zones balayées par les eaux vives, des prescriptions concernant les entrées piétonnes, les parkings souterrains, les ouvrages techniques de distribution électrique, la pression des eaux, la possibilité d'évacuation des rez-de-chaussée vers les étages supérieurs, etc... sont édictées.

Secteur 3: la Plaine.

Les niveaux de sous faces de planchers sont fixés par rapport au T.N.

Les voiries et parkings doivent coller au T.N.

De plus, un bassin de rétention de 100 litres par mètre carré imperméabilisé est exigé.

Il est bien évident que ce lourd dossier complexe est éminemment évolutif et modulé en permanence en fonction des différentes opportunités, urbanistiques, etc... 
HYDRAULIQUE PLUVIALE À NÎMES

Caractéristiques des bassins de retenue et carrières.

\begin{tabular}{|c|c|c|c|c|c|c|c|}
\hline AMONT & $\begin{array}{l}\mathrm{N}^{\circ} \text { du } \\
\text { bassin }\end{array}$ & $\begin{array}{l}\text { Volume } \\
\text { stocké }\left(\mathrm{m}^{3}\right)\end{array}$ & $\begin{array}{c}\text { Surface } \\
\text { mouillée }\left(\mathrm{m}^{2}\right)\end{array}$ & $\begin{array}{l}\text { Hauteur } \\
\text { digue }\end{array}$ & \multicolumn{2}{|c|}{$\begin{array}{c}\text { Surface du bassin } \\
\text { versant }\end{array}$} & Type \\
\hline \multicolumn{8}{|l|}{ LA PONDRE } \\
\hline Caveirac & 1 & 5700000 & 137500 & - & \multicolumn{2}{|c|}{460 ha } & C 5 \\
\hline VALDEGOUR & & & + & & & & \\
\hline Pierre Blanche & 2 & 40000 & 12350 & $8.4 \mathrm{~m}$ & \multicolumn{2}{|c|}{276 ha } & 1 \\
\hline Moure Froid & 3 & 39000 & 12800 & $9,3 \mathrm{~m}$ & \multicolumn{2}{|c|}{360 ha } & 1 \\
\hline \multicolumn{8}{|l|}{ CAMPLANIER } \\
\hline Vacquevrolles amont & 4 & 27700 & 31100 & $3.3 \mathrm{~m}$ & \multicolumn{2}{|c|}{220 ha } & R 1 \\
\hline Vacquevrolles aval & 5 & 54400 & 44000 & $5,2 \mathrm{~m}$ & \multicolumn{2}{|c|}{230 ha } & $\mathrm{R} 1$ \\
\hline Pont de la Rèpublique & 6 & 23700 & 10000 & $4 \mathrm{~m}$ & \multicolumn{2}{|c|}{540 ha } & R 1 \\
\hline \multicolumn{8}{|l|}{ ALES } \\
\hline Anduze & 7 & 20000 & 30000 & $3,5 \mathrm{~m}$ & \multicolumn{2}{|c|}{461 ha } & 3 \\
\hline Antiquailles & 8 & 3000000 & 90000 & & \multicolumn{2}{|c|}{461 ha } & 5 \\
\hline Roquemaillère & 9 & 40900 & 16700 & $7,3 \mathrm{~m}$ & \multicolumn{2}{|c|}{663 ha } & 1 \\
\hline Stade du Vallon $\mathrm{N}^{\circ} 1$ & 10 & 18000 & 7700 & $2,5 \mathrm{~m}$ & \multicolumn{2}{|c|}{149 ha } & C 3 \\
\hline Stade du Vallon $\mathrm{N}^{\circ} 2$ & 11 & 18500 & 10000 & $2,3 \mathrm{~m}$ & \multicolumn{2}{|c|}{149 ha } & 3 \\
\hline \multicolumn{8}{|l|}{ UZES } \\
\hline Terrain de larmée & 12 & 19800 & 12000 & $7,1 \mathrm{~m}$ & \multicolumn{2}{|c|}{66 ha } & 2 \\
\hline Engance & 13 & 1000 & 2000 & $2 \mathrm{~m}$ & \multicolumn{2}{|c|}{185 ha } & 4 \\
\hline Tennis de Calvas & 14 & 15300 & 9400 & $10,3 \mathrm{~m}$ & & & 1 \\
\hline Oliveraie & 15 & 94400 & 30000 & $11.6 \mathrm{~m}$ & & & 1 \\
\hline VALLAT RIQUET & & & & & & & \\
\hline Vallat Riquet & 16 & 32000 & 13000 & $6,1 \mathrm{~m}$ & & & R 1 \\
\hline VALLADAS & & & & & & & \\
\hline Grand Serre & 17 & 47000 & 17000 & $8 \mathrm{~m}$ & & & 2 \\
\hline Cimetière de Courbessac & 18 & 1000 & 3000 & $2,5 \mathrm{~m}$ & & & 4 \\
\hline Caravanes & 19 & 4000 & 2500 & $3 m$ & & & R 4 \\
\hline Aérodrome & 20 & 160000 & 200000 & $3 \mathrm{~m}$ & & & R3 \\
\hline CHILONNE & & & & & & & \\
\hline Baracine & 21 & 100000 & 28500 & $5.8 \mathrm{~m}$ & & & 1 \\
\hline TOTAL & & 9456700 & 719550 & & $\mathrm{R}=$ Réalise & $\mathrm{C}=\mathrm{En}$ cour & \\
\hline AVAL & $\begin{array}{l}\mathrm{N} d u \\
\text { bassin }\end{array}$ & $\begin{array}{c}\text { Volume } \\
\text { stockè }\left(\mathrm{m}^{3}\right)\end{array}$ & $\begin{array}{c}\text { Surface } \\
\text { mouilleee }\left(\mathrm{m}^{2}\right)\end{array}$ & $\begin{array}{l}\text { Hauteur } \\
\text { digue }\end{array}$ & $\begin{array}{r}\text { Sur } \\
\text { de } \\
\text { de la } \\
\end{array}$ & $\begin{array}{l}\text { S inondées et } \\
\text { bmersion en } \\
\text { ode de retour }\end{array}$ & $\begin{array}{l}\text { eurs } \\
\text { on } \\
\text { crue }\end{array}$ \\
\hline SAINT-CESAIRE & & & & & 10 ans & 40 ans & 100 ans \\
\hline Friches & 22 & 50000 & 36000 & 2 & $\begin{array}{c}\mathrm{S}=2,5 \mathrm{ha} \\
\mathrm{h}=1 \mathrm{~m}\end{array}$ & $\begin{array}{l}\mathrm{S}=2.7 \mathrm{ha} \\
\mathrm{h}=1.10 \mathrm{~m}\end{array}$ & $\begin{array}{c}\mathrm{S}=3.6 \mathrm{ha} \\
\mathrm{h}=2 \mathrm{~m}\end{array}$ \\
\hline Friches & 23 & 40000 & 24000 & 2 & $\begin{array}{l}\mathrm{S}=2,2 \mathrm{ha} \\
\mathrm{h}=1,60 \mathrm{~m}\end{array}$ & $\begin{array}{l}S=2,3 \text { ha } \\
h=1,70 \mathrm{~m}\end{array}$ & $\begin{array}{c}\mathrm{S}=2,4 \mathrm{ha} \\
\mathrm{h}=2 \mathrm{~m}\end{array}$ \\
\hline Friches + maraichers & 24 & 50000 & 36000 & 2 & $\begin{array}{l}\mathrm{S}=3,3 \mathrm{ha} \\
\mathrm{h}=1,60 \mathrm{~m}\end{array}$ & $\begin{array}{l}S=3,4 h a \\
h=1,70 \mathrm{~m}\end{array}$ & $\begin{array}{c}\mathrm{S}=3,5 \mathrm{ha} \\
\mathrm{h}=2 \mathrm{~m}\end{array}$ \\
\hline Vignes + blè & 25 & 40000 & 24000 & 2 & $\begin{array}{l}S=2,1 \text { ha } \\
h=1,50 \mathrm{~m}\end{array}$ & $\begin{array}{l}S=2,2 \text { ha } \\
h=1,60 \mathrm{~m}\end{array}$ & $\begin{array}{c}S=2,4 \text { ha } \\
h=2 \mathrm{~m}\end{array}$ \\
\hline VALDEGOUR & & & & & & & \\
\hline Centre auto-routier & 26 & 10000 & 35000 & 0,3 & & & $\begin{array}{l}S=3,5 \mathrm{ha} \\
\mathrm{h}=0,30 \mathrm{~m}\end{array}$ \\
\hline Tournesol & 27 & 55000 & 30000 & 2 & & $\begin{array}{l}\mathrm{S}=2,2 \mathrm{ha} \\
\mathrm{h}=0,85 \mathrm{~m}\end{array}$ & $\begin{array}{l}\mathrm{S}=3 \mathrm{ha} \\
\mathrm{h}=2 \mathrm{~m}\end{array}$ \\
\hline Tournesol & 28 & 130000 & 75000 & 2 & $\begin{array}{l}S=6,1 \text { ha } \\
h=0,60 \mathrm{~m}\end{array}$ & $\begin{array}{l}\mathrm{S}=6,6 \mathrm{ha} \\
\mathrm{h}=1,05 \mathrm{~m}\end{array}$ & $\begin{array}{c}\mathrm{S}=7.5 \mathrm{ha} \\
\mathrm{h}=2 \mathrm{~m}\end{array}$ \\
\hline ALES & & & & & & & \\
\hline Parking Stade & 29 & 25000 & 37000 & 0.3 & & $\begin{array}{l}\mathrm{S}=8,5 \mathrm{ha} \\
\mathrm{h}=0,25 \mathrm{~m}\end{array}$ & $\begin{array}{l}\mathrm{S}=8,7 \mathrm{ha} \\
\mathrm{h}=0,30 \mathrm{~m}\end{array}$ \\
\hline Pommiers & 30 & 170000 & 106000 & 2 & $\begin{array}{l}S=6,3 \mathrm{ha} \\
\mathrm{h}=0,45 \mathrm{~m}\end{array}$ & $\begin{array}{c}\mathrm{S}=9 \mathrm{ha} \\
\mathrm{h}=1,30 \mathrm{~m}\end{array}$ & $\begin{array}{c}\mathrm{S}=10,5 \mathrm{ha} \\
\mathrm{h}=2 \mathrm{~m}\end{array}$ \\
\hline UZES & & & & & & & \\
\hline Pommiers & 31 & 30000 & 23000 & 2 & & $\begin{array}{l}S=0,6 \text { ha } \\
h=0,20 \mathrm{~m}\end{array}$ & $\begin{array}{c}\mathrm{S}=2,3 \mathrm{ha} \\
\mathrm{h}=2 \mathrm{~m}\end{array}$ \\
\hline Pommiers & 32 & 15000 & 15000 & 2 & & $S=1,1$ ha & $\mathrm{S}=1,5 \mathrm{ha}$ \\
\hline VISTRE FONTAINE & & & & & & & \\
\hline Blé et maraichers & 33 & 50000 & 45000 & 2 & & $\begin{array}{l}\mathrm{S}=4 \mathrm{ha} \\
\mathrm{h}=1,4 \mathrm{~m}\end{array}$ & $\begin{array}{c}\mathrm{S}=4.5 \mathrm{ha} \\
\mathrm{h}=2 \mathrm{~m}\end{array}$ \\
\hline VALLADAS & & & & & & & \\
\hline Blé & 34 & 120000 & 73000 & 2 & & $\begin{array}{c}\mathrm{S}=6 \mathrm{ha} \\
\mathrm{h}=0,40 \mathrm{~m}\end{array}$ & $\begin{array}{c}\mathrm{S}=7,3 \mathrm{ha} \\
\mathrm{h}=2 \mathrm{~m}\end{array}$ \\
\hline Blé, tournesol et asperges & 35 & 120000 & 80000 & 2 & $\begin{array}{l}S=4,2 \text { ha } \\
h=0,25 \mathrm{~m}\end{array}$ & $\begin{array}{l}\mathrm{S}=7 \mathrm{ha} \\
\mathrm{h}=1,3 \mathrm{~m}\end{array}$ & $\begin{array}{l}\mathrm{S}=8 \mathrm{ha} \\
\mathrm{h}=2 \mathrm{~m}\end{array}$ \\
\hline Tournesol et friches & 36 & 40000 & 30000 & 2 & $\begin{array}{c}S=2 h a \\
h=0,80 m\end{array}$ & $\begin{array}{l}\mathrm{S}=2,5 \mathrm{ha} \\
\mathrm{h}=1,5 \mathrm{~m}\end{array}$ & $\begin{array}{l}\mathrm{S}=3 \mathrm{ha} \\
\mathrm{h}=2 \mathrm{~m}\end{array}$ \\
\hline Friches et prairie & 37 & 130000 & 75000 & 2 & $\begin{array}{l}\mathrm{S}=6,4 \mathrm{ha} \\
\mathrm{h}=0,80 \mathrm{~m}\end{array}$ & $\begin{array}{c}\mathrm{S}=7 \mathrm{ha} \\
\mathrm{h}=1,50 \mathrm{~m}\end{array}$ & $\begin{array}{c}\mathrm{S}=7.6 \mathrm{ha} \\
\mathrm{h}=2 \mathrm{~m}\end{array}$ \\
\hline TOTAL & & 1085000 & 800000 & & & & \\
\hline
\end{tabular}

